\title{
On minimal sets of graded attribute implications
}

\author{
Vilem Vychodil* \\ Dept. Computer Science, Palacky University, Olomouc
}

\begin{abstract}
We explore the structure of non-redundant and minimal sets consisting of graded if-then rules. The rules serve as graded attribute implications in object-attribute incidence data and as similarity-based functional dependencies in a similarity-based generalization of the relational model of data. Based on our observations, we derive a polynomial-time algorithm which transforms a given finite set of rules into an equivalent one which has the least size in terms of the number of rules.
\end{abstract}

\section{Introduction}

Reasoning with various types of if-then rules is crucial in many disciplines ranging from theoretical computer science to applications. Among the most widely used rules are those taking from of implications between conjunctions of attributes. Such rules are utilized in database systems (as functional dependencies or inclusion dependencies [23]), logic programming (as particular definite clauses representing programs [22]), and data mining (as attribute implications [14] or association rules [1, 33]). One of the most important problems regarding the rules is to find for a given set $T$ of rules a set of rules which is equivalent to $T$ and minimal in terms of its size. In relational database theory [23, the problem is referred to as finding minimal covers of $T$.

*e-mail: vychodil@binghamton.edu, phone: +420 585634 705, fax: +420585411643 
In this paper, we deal with the problem of finding minimal and equivalent sets of rules for general rules describing dependencies between graded attributes. That is, instead of the classic rules which are often considered as implications

$$
\left\{y_{1}, \ldots, y_{m}\right\} \Rightarrow\left\{z_{1}, \ldots, z_{n}\right\}
$$

between sets of attributes, describing presence/absence of attributes, we deal with rules where the presence/absence of attributes is expressed to degrees. That is, the rules in question can be written as

$$
\left\{{ }^{a_{1}} / y_{1}, \ldots,{ }^{a_{m}} / y_{m}\right\} \Rightarrow\left\{b^{b_{1}} / z_{1}, \ldots,{ }^{b_{n}} / z_{n}\right\}
$$

and understood as rules saying that "if $y_{1}$ is present at least to degree $a_{1}$ and $\cdots$ and $y_{m}$ is present at least to degree $a_{m}$, then $z_{1}$ is present at least to degree $b_{1}$ and $\cdots$ and $z_{n}$ is present at least to degree $b_{n}$." We assume that the degrees appearing in 22 come from a structure of truth degrees which is more general than the two-element Boolean algebra and allows for intermediate degrees of truth. In particular, we use complete residuated lattices [13] with linguistic hedges [12, 19, 29] for the job. In our setting, (2) can be seen as generalization of (1) if all the degrees $a_{1}, \ldots, b_{1}, \ldots$ are equal to 1 (as usual, 1 denotes the classical truth value of "full truth").

Our previous results on rules of the form (2) include a fixed point characterization of a semantic entailment, Armstrong-style [2] axiomatizations in the ordinary style and the graded style (also known as Pavelka-style completeness, see $[24,25,26]$ ), results on generating non-redundant bases from data, and two kinds of semantics of the rules: (i) a database semantics which is based on evaluating the rules in ranked data tables over domains with similarities [5], and (ii) an incidence data semantics which is based on evaluating the rules in objectattribute data tables with graded attributes [4, 7] which are known as formal contexts in formal concept analysis [14. Analogously as for the ordinary rules, one can show that both (i) and (ii) yield the same notion of semantic entailment which simplifies further considerations, e.g., a single axiomatization of the se- 
mantic entailment works for both the database and incidence data semantics of the rules. A survey of recent results regarding the rules can be found in 8 .

In this paper, we consider rules like (2) and explore the structure of nonredundant and minimal sets of rules of this type. We show an if-and-only-if criterion of minimality and a polynomial-time procedure which, given $T$, transforms $T$ into an equivalent and minimal set of graded rules. Let us note that the previous results regarding minimality of sets of graded rules $[8]$ were focused exclusively on sets of rules generated from data. That is, the input for such instance-based approaches is not a set $T$ of rules. Instead, the input is assumed to be a structure (e.g., a formal context with graded attributes or a database table over domains with similarities) and the goal is to find a minimal set $T$ of rules which entails exactly all the rules true in the structure. One particular example is an algorithm for generating graded counterparts to Guigues-Duquenne bases [17] described in [8]. In contrast, the problem studied in this paper is different. We assume that a set $T$ of rules is already given (e.g., inferred from data or proposed by an expert) but it may not be minimal. Therefore, it is interesting to find a minimal set of rules which conveys the same information. Unlike the instance-based methods which belong to hard problems [10] even for the classic (non-graded) rules, the minimization method presented in this paper is polynomial and therefore tractable.

The present paper is organized as follows. Section 2 presents preliminaries from structures of degrees and graded if-then rules. Section 3 contains the new results.

\section{Preliminaries}

In this section, we present basic notions from structures of truth degrees and graded attribute implications which formalize rules like (2). We only present the notions and results which are sufficient to follow the results in Section 3 Interested readers may find more results in 4, , 8, 13, 15, 18, 20.

A (complete) residuated lattice [4, 13] is an algebra $\mathbf{L}=\langle L, \wedge, \vee, \otimes, \rightarrow$ 
$, 0,1\rangle$ where $\langle L, \wedge, \vee, 0,1\rangle$ is a (complete) lattice, $\langle L, \otimes, 1\rangle$ is a commutative monoid, and $\otimes$ (multiplication, a truth function of "fuzzy conjunction") and $\rightarrow$ (residuum, a truth function of "fuzzy implication") satisfy the adjointness property: $a \otimes b \leq c$ iff $a \leq b \rightarrow c(a, b, c \in L)$. Examples of complete residuated lattices include structures on the real unit interval given by left-continuous t-norms [11, 18] as well as finite structures of degrees.

If $U \neq \emptyset$, we can consider the direct power $\mathbf{L}^{U}=\left\langle L^{U}, \cap, \cup, \otimes, \rightarrow,{ }^{*}, \emptyset_{U}, 1_{U}\right\rangle$ of $\mathbf{L}$. Each $A \in L^{U}$ is called an $\mathbf{L}$-set (s fuzzy set) $A$ in universe $U$. That is, $A \in L^{U}$ is a map $A: U \rightarrow L, A(u)$ being interpreted as "the degree to which $u$ belongs to $A$ ". Operations $\cap, \cup, \otimes, \ldots$ in $\mathbf{L}^{U}$ represent operations with $\mathbf{L}$-sets which are induced by the corresponding operations $\wedge, \vee, \otimes, \ldots$ in $\mathbf{L}$. Hence, e.g., $(A \cup B)(u)=A(u) \vee B(u)$ for each $u \in U$. Note that for the lattice order $\subseteq$ in $\mathbf{L}^{U}$ being induced by $\leq$, we have $A \subseteq B$ iff, for each $u \in U, A(u) \leq B(u)$. Therefore, $A \subseteq B$ denotes "full containment" of $A$ in $B$. If $U=\left\{u_{1}, \ldots, u_{n}\right\}$ ( $U$ is finite), we adopt the usual conventions for writing $\mathbf{L}$-sets $A \in L^{U}$ as $\left\{{ }^{a_{1}} / u_{1}, \ldots,{ }^{a_{n}} / u_{n}\right\}$ meaning that $A\left(u_{i}\right)=a_{i}(i=1, \ldots, n)$. Furthermore, in the notation we omit ${ }^{a_{i}} / u_{i}$ if $a_{i}=0$ and write $u_{i}$ if $a_{i}=1$.

Let $Y$ be a finite non-empty set of attributes (i.e., symbolic names). A graded attribute implication in $Y$ is an expression $A \Rightarrow B$, where $A, B \in L^{Y}$. In our paper, graded attribute implications are regarded as formulas representing rules like (2). The interpretation of graded attribute implications is based on the notion of a graded subsethood of $\mathbf{L}$-sets in a similar way as the interpretation of the ordinary attribute implications $[14$ is based on the ordinary subsethood. In a more detail, for any $A, M \in L^{Y}$, we define a degree $S(A, M) \in L$ of subsethood of $A$ in $M$ by

$$
S(A, M)=\bigwedge_{y \in Y}(A(y) \rightarrow M(y)) .
$$

Clearly, $A \subseteq M$ (i.e., $A$ is fully contained in $M$ ) iff $S(A, M)=1$. For any 
$A, B, M \in L^{Y}$, we may put

$$
\|A \Rightarrow B\|_{M}= \begin{cases}S(B, M), & \text { if } A \subseteq M \\ 1, & \text { otherwise }\end{cases}
$$

and call $\|A \Rightarrow B\|_{M}$ a degree to which $A \Rightarrow B$ is true in $M$. Therefore, if $M$ is regarded as an $\mathbf{L}$-set of attributes of an object with each $M(y)$ interpreted as the degree to which the object has attribute $y$, then $\|A \Rightarrow B\|_{M}$ is a degree to which the following statement is true: "If the object has all the attributes from $A$, then it has all the attributes from $B$ ". Interestingly, (4) is not the only possible (and reasonable) interpretation of $A \Rightarrow B$ in $M$. In fact, our approach in 8 ] is more general in that it defines $\|A \Rightarrow B\|_{M}^{*}$ by

$$
\|A \Rightarrow B\|_{M}^{*}=S(A, M)^{*} \rightarrow S(B, M)
$$

where $^{*}$ is an idempotent truth-stressing linguistic hedge [29, 30, 31, 32] on $\mathbf{L}$ (shortly, a hedge). We assume that ${ }^{*}$ is a map ${ }^{*}: L \rightarrow L$ such that (i) $1^{*}=1$, (ii) $a^{*} \leq a$, (iii) $(a \rightarrow b)^{*} \leq a^{*} \rightarrow b^{*}$, and (iv) $a^{* *}=a^{*}(a, b \in L)$. A hedge * can be seen as a generalization of Baaz's $\Delta$ operation [3, 18, and it has been introduced in fuzzy logic in the narrow sense [16] by Hájek in [19]. In the sense of [19], * can be seen as a truth function for unary logical connective "very true".

Now, one can see that (4) is a particular case of (5) for * being the so-called globalization [27]:

$$
a^{*}= \begin{cases}1, & \text { if } a=1, \\ 0, & \text { otherwise. }\end{cases}
$$

Indeed, for * introduced by (6), we have either $a^{*} \rightarrow b=1$ if $a<1$ or $a^{*} \rightarrow b=b$ if $a=1$ and thus (5) becomes (4). Note that in case of linearly ordered structures of truth degrees, globalization coincides with Baaz's $\Delta$ operation (this is not true in general). On the other hand, if * is identity, then the right-hand side of (5) becomes

$$
S(A, M) \rightarrow S(B, M)
$$

which may also be regarded as a desirable interpretation of $A \Rightarrow B$ in $M$. The approach via hedges in [8] allows us to deal with both (4) and (7) (and possibly 
other interpretations resulting by the choice of other hedges) in a unified way because (4) and (7) result as two borderline choices of hedges in (5), namely, the globalization and the identity on $L$. Also, since * may be interpreted as a truth function of logical connective "very true", the general degree $\| A \Rightarrow$ $B \|_{M}^{*}$ introduced by (5) may be interpreted as a truth degree of the following statement: "If it is very true that the object (whose attributes are represented by $M$ ) has all the attributes from $A$, then it has all the attributes from $B$ ". Therefore, we may view the hedge as a parameter of the interpretation of graded attribute implications, see [8] for a detailed explanation and further remarks on the role of hedges. Recent results on hedges and their treatment in fuzzy logics in the narrow sense can be found in [12].

For graded attribute implications, we introduce a semantic and a syntactic entailment (a provability) as follows. A set $T$ of graded attribute implications (in $Y$ ) is called a theory (in $Y$ ). An L-set $M \in L^{Y}$ is called a model of $T$ if $\|A \Rightarrow B\|_{M}^{*}=1$ for all $A \Rightarrow B \in T$. Let $\operatorname{Mod}(T)$ denote the set of all models of $T$. The degree $\|A \Rightarrow B\|_{T}^{*}$ to which $A \Rightarrow B$ is semantically entailed by $T$ is defined by

$$
\|A \Rightarrow B\|_{T}^{*}=\bigwedge_{M \in \operatorname{Mod}(T)}\|A \Rightarrow B\|_{M}^{*}
$$

Put in words, $\|A \Rightarrow B\|_{T}^{*}$ is a degree to which $A \Rightarrow B$ is true in all models of $T$. A graded attribute implication $A \Rightarrow B$ is called trivial whenever $\|A \Rightarrow B\|_{\emptyset}^{*}=1$.

The syntactic entailment of graded attribute implications is based on an Armstrong-style axiomatic system [2]. Namely, each $A \cup B \Rightarrow A\left(A, B \in L^{Y}\right)$ is considered as an axiom and we consider the following deduction rules [8]:

(Cut) from $A \Rightarrow B$ and $B \cup C \Rightarrow D$ infer $A \cup C \Rightarrow D$,

(Mul) from $A \Rightarrow B$ infer $c^{*} \otimes A \Rightarrow c^{*} \otimes B$,

where $A, B, C, D \in L^{Y}, c \in L$, and $c^{*} \otimes A$ (and analogously $c^{*} \otimes B$ ) denotes the so-called $c^{*}$-multiple of $A \in L^{Y}$ which is an $\mathbf{L}$-set such that $\left(c^{*} \otimes A\right)(y)=$ $c^{*} \otimes A(y)$ for all $y \in Y$. Note that in database literature, the classic counterpart 
to (Cut) is known under the name pseudo-transitivity, see [23]. The name cut comes from 21]. A proof of $A \Rightarrow B$ from $T$ is a sequence $\varphi_{1}, \ldots, \varphi_{n}$ such that $\varphi_{n}$ equals $A \Rightarrow B$ and for each $\varphi_{i}$ we either have $\varphi_{i} \in T$, or $\varphi_{i}$ is an axiom, or $\varphi_{i}$ is derived by (Cut) or (Mul) from some of $\varphi_{1}, \ldots, \varphi_{i-1}$. A graded attribute implication $A \Rightarrow B$ is provable from $T$, denoted $T \vdash A \Rightarrow B$ if there is a proof of $A \Rightarrow B$ from $T$. In the paper, we utilize properties of $\vdash$ called the additivity, projectivity, and transitivity, i.e., we use the facts that

(Add) $\{A \Rightarrow B, A \Rightarrow C\} \vdash A \Rightarrow B \cup C$,

(Pro) $\{A \Rightarrow B \cup C\} \vdash A \Rightarrow B$,

(Tra) $\{A \Rightarrow B, B \Rightarrow C\} \vdash A \Rightarrow C$.

for all $A, B, C \in L^{Y}$, see $[8$.

Remark 1 . Let us note that the trivial graded attribute implications are exactly the axioms, i.e., all graded attribute implications which are true in all models to degree 1 are of the form $A \cup B \Rightarrow A$. Also note that if * is (6), then (Mul) becomes a trivial deduction rule and can be disregarded. Let us stress that $\cup$ in the above expressions denotes the operation in $\mathbf{L}_{Y}$ induced by $\vee$ in $\mathbf{L}$. Therefore, the antecedent of $A \cup B \Rightarrow A$ should be read "the union of $A$ and $B$ ", etc.

For each $A \in L^{Y}$, the least model of $T$ which contains $A \in L^{Y}$ is called the (semantic) closure of $A$ and is denoted by $[A]_{T}$. For each $A \in L^{Y}$ and $T,[A]_{T}$ always exists since the set of all models of $T$ is closed under arbitrary intersections. The following ordinary-style [18] completeness theorem is established:

Theorem 1 (completeness, see [8]). Let $\mathbf{L}$ and $Y$ be finite. Then, for any $T$ and $A, B \in L^{Y}$, the following conditions are equivalent:

(i) $T \vdash A \Rightarrow B$,

(ii) $B \subseteq[A]_{T}$,

(iii) $\|A \Rightarrow B\|_{T}^{*}=1$. 
Taking into account Theorem 1, we may freely interchange the semantic entailment (to degree 1) and provability on condition that both $\mathbf{L}$ and $Y$ are finite which we assume from now on - cases of infinite $\mathbf{L}$ can be handled by adding an infinitary rule but the issue is not relevant to this paper, cf. [8]. Theory $T$ is non-redundant if $T \backslash\{A \Rightarrow B\} \nvdash A \Rightarrow B$ for all $A \Rightarrow B \in T$. Theories $T_{1}$ and $T_{2}$ are equivalent if, for all $A, B \in L^{Y}, T_{1} \vdash A \Rightarrow B$ iff $T_{2} \vdash A \Rightarrow B$.

Remark 2. (a) Alternative graph-based proof systems [28] as well as automated provers based on simplification equivalences as in [9] are also available. Let us also note that in addition to Theorem 1 which provides a syntactic characterization only for formulas which are semantically entailed to degree 1 , the logic of graded attribute implications is also complete in the graded style (Pavelka-style completeness). Namely, $\|A \Rightarrow B\|_{T}^{*}=\bigvee\{c \in L \mid T \vdash A \Rightarrow c \otimes B\}$, cf. $[8]$.

(b) The general interpretation of $A \Rightarrow B$ in $M$ introduced in (5) corresponds to the incidence data semantics we have mentioned in the introduction. There are alternative interpretations which yield the same notion of semantic entailment. For instance, instead of $M$, one may take (ranked) data tables over domains with similarities and define the interpretation of $A \Rightarrow B$ in such structures. In effect, the graded implications interpreted this way can be seen as similarity-based functional dependencies and play analogous role to the ordinary functional dependencies in the classical relational model of data. Since the database and incidence data semantics yield the same notions of semantic entailment and thus the same complete axiomatization, we refrain from commenting on further details. Interested readers may check $[8]$.

\section{Results}

Recall that procedures for removing redundancy from theories are well known $[8]$. That is, given a finite theory $T$, one may compute $T^{\prime} \subseteq T$ which is equivalent to $T$ and which is in addition non-redundant. Indeed, according to Theorem 1 
$T$ is redundant iff there is $A \Rightarrow B \in T$ such that $B \subseteq[A]_{T \backslash\{A \Rightarrow B\}}$ in which case one can remove $A \Rightarrow B$ from $T$ and repeat the procedure until $T$ becomes non-redundant. This procedure can be used to remove all formulas which make $T$ redundant but it does not guarantee that the result is minimal in terms of the number of formulas in $T$. In this paper, we show one approach to deal with the issue.

For practical reasons, we limit ourselves to finite theories. Otherwise, in general we would not be able to transform a theory into an equivalent and minimal one in finitely many steps. Furthermore, we assume that ${ }^{*}$ is globalization, i.e., $\|A \Rightarrow B\|_{M}^{*}$ is in fact given by (4) and (Mul) can be omitted. In the text, we give counterexamples indicating that the present theory cannot be directly generalized for general hedges at least not with a substantial modification. Interestingly, the instance-based approaches have an analogous practical limitation, cf. [8].

We start by presenting a technical observation on the properties of provability which also depends on * being the globalization.

Theorem 2. Let $T$ be a theory such that $T \vdash A \Rightarrow B$ and $T \backslash\{C \Rightarrow D\} \nvdash A \Rightarrow$ B. Then, $T \backslash\{C \Rightarrow D\} \vdash A \Rightarrow C$.

Proof. Observe that $T \vdash A \Rightarrow B$ and $T \backslash\{C \Rightarrow D\} \nvdash A \Rightarrow B$ means that each proof of $A \Rightarrow B$ by $T$ contains $C \Rightarrow D$. Using properties of closures, we get $B \nsubseteq[A]_{T \backslash\{C \Rightarrow D\}}, B \subseteq[A]_{T}$, and $[A]_{T \backslash\{C \Rightarrow D\}} \subseteq[A]_{T}$ which altogether yield $[A]_{T \backslash\{C \Rightarrow D\}} \subset[A]_{T}$. In order to prove $T \backslash\{C \Rightarrow D\} \vdash A \Rightarrow C$, it suffices to show that $C \subseteq[A]_{T \backslash\{C \Rightarrow D\}}$. By contradiction, assume that $C \nsubseteq[A]_{T \backslash\{C \Rightarrow D\}}$. By definition, $[A]_{T \backslash\{C \Rightarrow D\}}$ is the least model of $T \backslash\{C \Rightarrow D\}$ containing $A$. Now, observe that since ${ }^{*}$ is globalizaton, it follows that (5) becomes (4) and thus $\|C \Rightarrow D\|_{[A]_{T \backslash\{C \Rightarrow D\}}^{*}}^{*}=1$ because $C \nsubseteq[A]_{T \backslash\{C \Rightarrow D\}}$. As a consequence, $[A]_{T \backslash\{C \Rightarrow D\}} \in \operatorname{Mod}(T)$ because $T$ and $T \backslash\{C \Rightarrow D\}$ differ only in the presence of $C \Rightarrow D$ in $T$. Hence, $[A]_{T \backslash\{C \Rightarrow D\}}$ is a model of $T$ which contains $A$ and since $[A]_{T}$ is the least model of $T$ containing $A$, we must have $[A]_{T} \subseteq[A]_{T \backslash\{C \Rightarrow D\}}$, which contradicts the fact that $[A]_{T \backslash\{C \Rightarrow D\}} \subset[A]_{T}$. Therefore, we have $C \subseteq$ 
$[A]_{T \backslash\{C \Rightarrow D\}}$ which gives $T \backslash\{C \Rightarrow D\} \vdash A \Rightarrow C$.

Example 1. Let us show that Theorem 2 does not hold in the case of general hedges. For instance, let $\mathbf{L}$ be a three-element equidistant subchain of the standard Lukasiewicz algebra with ${ }^{*}$ being the identity. That is, $L=\{0,0.5,1\}$, $\wedge$ and $\vee$ coincide with maximum and minimum, respectively, and

$$
\begin{gathered}
a \otimes b=\max (0, a+b-1), \\
a \rightarrow b=\min (1,1-a+b),
\end{gathered}
$$

for all $a, b \in L$. Consider $T=\{\{y\} \Rightarrow\{z\}\}$. Obviously, $T \vdash\{0.5 / y\} \Rightarrow\{0.5 / z\}$ on account of (Mul). In addition, $\left\{{ }^{0.5} / y\right\} \Rightarrow\left\{{ }^{0.5} / z\right\}$ is non-trivial and thus not provable by the empty set of formulas. Furthermore, $T \nvdash\{0.5 / y\} \Rightarrow\{y\}$. Indeed, for $M=\left\{0.5 / y,{ }^{0.5} / z\right\}$, we get

$$
S(\{y\}, M)=(1 \rightarrow 0.5) \wedge(0 \rightarrow 0.5)=0.5 \wedge 1=0.5=S(\{z\}, M),
$$

i.e., $M \in \operatorname{Mod}(T)$. On the other hand,

$$
S(\{0.5 / y\}, M)=(0.5 \rightarrow 0.5) \wedge(0 \rightarrow 0.5)=1 \wedge 1=1 \not \leq 0.5=S(\{y\}, M),
$$

i.e., $\left\|\left\{{ }^{0.5} / y\right\} \Rightarrow\{y\}\right\|_{M}^{*}<1$. Hence, due to soundness, $T \nvdash\left\{\left\{^{0.5} / y\right\} \Rightarrow\{y\}\right.$ which illustrates that in case of general hedges, one cannot always conclude $T \backslash\{C \Rightarrow D\} \vdash A \Rightarrow C$ provided that $T \vdash A \Rightarrow B$ and $T \backslash\{C \Rightarrow D\} \nvdash A \Rightarrow B$.

As a consequence of Theorem 2, we can prove an assertion which gives us new insight into the structure of non-redundant theories. The assertion matches formulas from non-redundant theories based on the following notions of equivalence:

Definition 3. Let $T$ be a theory and $A, B \in L^{Y}$. We say that $A$ and $B$ are provably equivalent under $T$, written $A \equiv_{T} B$, whenever $T \vdash A \Rightarrow B$ and $T \vdash B \Rightarrow A$.

Obviously, $\equiv_{T}$ is an equivalence relation on $L^{Y}$. Using the notion, we establish the following observation. 
Theorem 4. Let $T_{1}$ and $T_{2}$ be equivalent and non-redundant. Then, for each $A \Rightarrow B \in T_{1}$ there is $C \Rightarrow D \in T_{2}$ such that $A \equiv_{T_{1}} C$.

Proof. Take $A \Rightarrow B \in T_{1}$. Since $T_{1}$ and $T_{2}$ are equivalent, we get $T_{2} \vdash A \Rightarrow B$. Therefore, there is a proof of $A \Rightarrow B$ by $T_{2}$ which uses finitely many formulas $\varphi_{1}, \ldots, \varphi_{k}$ in $T_{2}$. In addition, we can select a subset $T_{2}^{\prime} \subseteq\left\{\varphi_{1}, \ldots, \varphi_{k}\right\}$ such that $T_{2}^{\prime} \vdash A \Rightarrow B$ and $T^{\prime \prime} \nvdash A \Rightarrow B$ for all $T^{\prime \prime} \subset T_{2}^{\prime}$. Observe that since $A \Rightarrow B \in T_{1}$ and $T_{1}$ is non-redundant, then $A \Rightarrow B$ is non-trivial. Therefore, $T_{2}^{\prime}$ is non-empty. Clearly, we get $T_{1} \vdash \varphi$ for each $\varphi \in T_{2}^{\prime}$ since $T_{1}$ and $T_{2}$ are equivalent.

We now claim that there is $C \Rightarrow D \in T_{2}^{\prime}$ such that each proof of $C \Rightarrow D$ by $T_{1}$ contains $A \Rightarrow B$, i.e., $T_{1} \backslash\{A \Rightarrow B\} \nvdash C \Rightarrow D$. By contradiction, assume that $T_{1} \backslash\{A \Rightarrow B\} \vdash C \Rightarrow D$ for each $C \Rightarrow D \in T_{2}^{\prime}$. Since $T_{2}^{\prime} \vdash A \Rightarrow B$, we would get that $T_{1} \backslash\{A \Rightarrow B\} \vdash A \Rightarrow B$ which contradicts the fact that $T_{1}$ is non-redundant.

Finally, by $T_{2}^{\prime} \vdash A \Rightarrow B$ and $T_{2}^{\prime} \backslash\{C \Rightarrow D\} \nvdash A \Rightarrow B$, we get that $T_{2}^{\prime} \vdash A \Rightarrow C$ by Theorem 2 and thus $T_{1} \vdash A \Rightarrow C$. Moreover, $C \Rightarrow D \in T_{2}^{\prime}$ implies that $T_{1} \vdash C \Rightarrow D$. Using the fact that $T_{1} \backslash\{A \Rightarrow B\} \nvdash C \Rightarrow D$ and Theorem 2 we get $T_{1} \vdash C \Rightarrow A$. Altogether, $T_{1} \vdash A \Rightarrow C$ and $T_{1} \vdash C \Rightarrow A$ give $A \equiv_{T_{1}} C$.

Example 2. Let us consider the same structure of truth degrees as in Example1 and let $Y=\{x, y, z\}$. One can check that the following theories

$$
\begin{aligned}
T_{1}=\{ & \left.\left\{{ }^{0.5} / y\right\} \Rightarrow\left\{x,{ }^{0.5} / y, z\right\},\{z\} \Rightarrow\left\{{ }^{0.5} / x\right\},\{x, z\} \Rightarrow\left\{{ }^{0.5} / x, y,{ }^{0.5} / z\right\}\right\}, \\
T_{2}=\{ & \{z\} \Rightarrow\left\{{ }^{0.5} / x\right\},\left\{{ }^{0.5} / x, y,{ }^{0.5} / z\right\} \Rightarrow\left\{x,{ }^{0.5} / y,{ }^{0.5} / z\right\} \\
& \left\{{ }^{0.5} / y\right\} \Rightarrow\left\{{ }^{0.5} / x,{ }^{0.5} / y, z\right\},\left\{^{0.5} / x,{ }^{0.5} / y\right\} \Rightarrow\{y, z\}, \\
& \left.\{x, z\} \Rightarrow\left\{^{0.5} / x, y,{ }^{0.5} / z\right\}\right\}
\end{aligned}
$$

are non-redundant and equivalent. Indeed, one can check the fact either by showing that each formula in $T_{1}$ is provable by $T_{2}$ and vice versa which is straightforward but tedious, or one can show that both $T_{1}$ and $T_{2}$ have the 


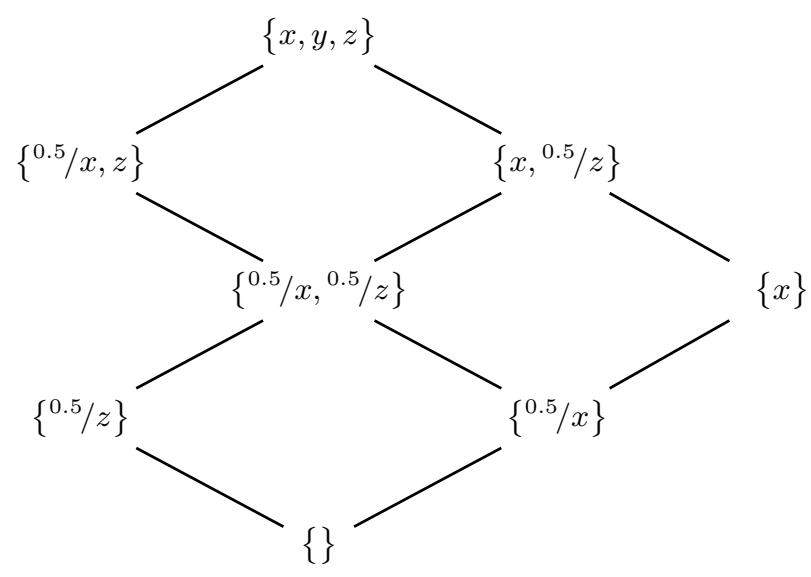

Figure 1: Lattice of models of theories $T_{1}$ and $T_{2}$ from Example 1

same models. Figure 1 shows the set of all models of either of the theories ordered by the inclusion of $\mathbf{L}$-sets.

Now, for each formula in $T_{1}$ there is a formula in $T_{2}$ which has the same antecedent. Thus, in this direction, the consequence of Theorem 4 is immediate. On the other hand, for $\left\{0.5 / x,{ }^{0.5} / y\right\} \Rightarrow\{y, z\} \in T_{2}$ there is no formula in $T_{1}$ with the antecedent equal to $\left\{{ }^{0.5} / x,{ }^{0.5} / y\right\}$. Nevertheless, we have

$$
\begin{aligned}
& \{.5 / x, 0.5 / y\} \equiv_{T_{2}}\left\{{ }^{0.5} / y\right\} \\
& \left\{0.5 / x,{ }^{0.5} / y\right\} \equiv_{T_{2}}\{x, z\}
\end{aligned}
$$

i.e., one can take $\{0.5 / y\} \Rightarrow\left\{x,{ }^{0.5} / y, z\right\} \in T_{1}$ or $\{x, z\} \Rightarrow\{0.5 / x, y, 0.5 / z\} \in T_{1}$ and the same applies to $\left\{0.5 / x, y,{ }^{0.5} / z\right\} \Rightarrow\{x, 0.5 / y, 0.5 / z\} \in T_{2}$.

As in Example 1, it can be shown that Theorem 4 cannot be extended to general hedges. For instance, consider the following theories

$$
\begin{aligned}
& T_{3}=\{\{\} \Rightarrow\{y, 0.5 / z\}\}, \\
& T_{4}=\left\{\left\{^{0.5} / y\right\} \Rightarrow\{y\},\{z\} \Rightarrow\left\{^{0.5} / y,{ }^{0.5} / z\right\},\{\} \Rightarrow\left\{^{0.5} / z\right\}\right\},
\end{aligned}
$$

and let $\mathbf{L}$ be the three-element Gödel chain with * being the identity. That is, 
$\mathbf{L}$ is defined as in Example 1 except that $\otimes$ coincides with $\wedge$,

$$
a \rightarrow b= \begin{cases}1, & \text { if } a \leq b, \\ b, & \text { otherwise }\end{cases}
$$

and $0.5^{*}=0.5$. In this setting, $T_{3}$ and $T_{4}$ are both non-redundant and equivalent. Now, for $\{z\} \Rightarrow\left\{0.5 / y,{ }^{0.5} / z\right\} \in T_{4}$ there is no formula in $T_{3}$ whose antecedent is equivalent to $\{z\}$. Indeed, $T_{3} \nvdash\{\} \Rightarrow\{z\}$ on account of soundness because $\{y, 0.5 / z\}$ is a model of $T_{3}$.

The relationship between formulas based on equivalence of their antecedents is crucial for our investigation. We therefore introduce the following notation. For each $A \in L^{Y}$ and theory $T$, put

$$
\mathrm{E}_{T}(A)=\left\{C \Rightarrow D \in T \mid A \equiv_{T} C\right\}
$$

i.e., $\mathrm{E}_{T}(A)$ is a subset of $T$ containing all formulas whose antecedent is equivalent to $A$. For particular $A$ and $T$, we may have $\mathrm{E}_{T}(A)=\emptyset$. The collection of all non-empty subsets of the form (9) for $A \in L^{Y}$ is obviously a partition of $T$. We denote the partition by $\mathcal{E}_{T}$, i.e.,

$$
\mathcal{E}_{T}=\left\{\mathrm{E}_{T}(A) \mid \mathrm{E}_{T}(A) \neq \emptyset \text { and } A \in L^{Y}\right\} .
$$

The partitions $(10)$ and their classes $(9)$ are illustrated by the following example.

Example 3. Consider theories $T_{1}$ and $T_{2}$ from Example 2 and the structure of degrees considered therein. In case of $T_{1}$, the partition $\mathcal{E}_{T_{1}}$ given by 10 consists of two distinct subsets of $T_{1}$. Namely,

$$
\mathcal{E}_{T_{1}}=\left\{\left\{\{0.5 / y\} \Rightarrow\{x, 0.5 / y, z\},\{x, z\} \Rightarrow\left\{{ }^{0.5} / x, y,{ }^{0.5} / z\right\}\right\},\{\{z\} \Rightarrow\{0.5 / x\}\}\right\} .
$$

In case of $T_{2}$, we get:

$$
\begin{aligned}
\mathcal{E}_{T_{2}}=\{ & \left\{\left\{{ }^{0.5} / x, y,{ }^{0.5} / z\right\} \Rightarrow\left\{x,{ }^{0.5} / y,{ }^{0.5} / z\right\},\left\{{ }^{0.5} / y\right\} \Rightarrow\left\{{ }^{0.5} / x,{ }^{0.5} / y, z\right\},\right. \\
& \left.\left.\left\{{ }^{0.5} / x,{ }^{0.5} / y\right\} \Rightarrow\{y, z\},\{x, z\} \Rightarrow\left\{{ }^{0.5} / x, y,{ }^{0.5} / z\right\}\right\},\left\{\{z\} \Rightarrow\left\{{ }^{0.5} / x\right\}\right\}\right\} .
\end{aligned}
$$

Observe that $\mathrm{E}_{T_{1}}(\{z\})=\mathrm{E}_{T_{2}}(\{z\})$ whereas $\mathrm{E}_{T_{1}}(\{x, z\}) \neq \mathrm{E}_{T_{2}}(\{x, z\})$. 
Remark 3. Obviously, if $A \equiv_{T} B$, then $\mathrm{E}_{T}(A)=\mathrm{E}_{T}(B)$. Conversely, if $\mathrm{E}_{T}(A)=$ $\mathrm{E}_{T}(B) \neq \emptyset$, then there is $C \Rightarrow D \in \mathrm{E}_{T}(A)=\mathrm{E}_{T}(B)$ and thus $A \equiv_{T} C$ and $B \equiv_{T} C$, i.e., we get $A \equiv_{T} B$. Note that the assumption on $\mathrm{E}_{T}(A)$ and $\mathrm{E}_{T}(B)$ being non-empty used in the latter claim cannot be dropped. For instance, for $T=\emptyset$ and $A, B \in L^{Y}$ such that $A \nsubseteq B$ and $B \nsubseteq A$, we get $\mathrm{E}_{T}(A)=\mathrm{E}_{T}(B)=\emptyset$ and $\emptyset \nvdash A \Rightarrow B$.

The following assertions show that despite the fact that equivalent nonredundant theories can differ in their size, the corresponding partitions 10 have always the same number of classes.

Theorem 5. Let $T_{1}$ and $T_{2}$ be equivalent and non-redundant. Then for any $A, B \in L^{Y}$, the following conditions hold:

(i) If $\mathrm{E}_{T_{1}}(A) \neq \emptyset$, then $\mathrm{E}_{T_{2}}(A) \neq \emptyset$.

(ii) If $\mathrm{E}_{T_{1}}(A)=\mathrm{E}_{T_{1}}(B) \neq \emptyset$, then $\mathrm{E}_{T_{2}}(A)=\mathrm{E}_{T_{2}}(B) \neq \emptyset$.

Proof. In order to prove (i), observe that if $\mathrm{E}_{T_{1}}(A) \neq \emptyset$ for $A \in L^{Y}$, then there is $C \Rightarrow D \in T_{1}$ such that $A \equiv_{T_{1}} C$. Theorem 4 yields there is $G \Rightarrow H \in T_{2}$ such that $C \equiv_{T_{1}} G$. Since $\equiv_{T_{1}}$ is transitive, we get $A \equiv_{T_{1}} G$. As a consequence, $A \equiv_{T_{2}} G$ because $T_{1}$ and $T_{2}$ are equivalent. Therefore, $G \Rightarrow H \in \mathrm{E}_{T_{2}}(A)$ and so we have $\mathrm{E}_{T_{2}}(A) \neq \emptyset$.

Now, (ii) is a consequence of (i) and the argument in Remark 3 Indeed, if $\mathrm{E}_{T_{1}}(A)=\mathrm{E}_{T_{1}}(B) \neq \emptyset$, then $A \equiv_{T_{1}} B$ and so $A \equiv_{T_{2}} B$ because $T_{1}$ and $T_{2}$ are equivalent. As a consequence, $\mathrm{E}_{T_{2}}(A)=\mathrm{E}_{T_{2}}(B) \neq \emptyset$ on account of (i).

Corollary 6. Let $T_{1}$ and $T_{2}$ be equivalent and non-redundant theories. Then, $\left|\mathcal{E}_{T_{1}}\right|=\left|\mathcal{E}_{T_{2}}\right|$.

Proof. Theorem 5 allows us to consider a map $h: \mathcal{E}_{T_{1}} \rightarrow \mathcal{E}_{T_{2}}$ which is defined by $h\left(\mathrm{E}_{T_{1}}(A)\right)=\mathrm{E}_{T_{2}}(A)$, for each $A \in L^{Y}$ such that $\mathrm{E}_{T_{1}}(A) \neq \emptyset$. Indeed, Theorem 5 ensures that the map is well defined. In addition, the map is injective because $h\left(\mathrm{E}_{T_{1}}(A)\right)=h\left(\mathrm{E}_{T_{1}}(B)\right)$ means $\mathrm{E}_{T_{2}}(A)=\mathrm{E}_{T_{2}}(B) \neq \emptyset$ and so $\mathrm{E}_{T_{1}}(A)=\mathrm{E}_{T_{1}}(B)$ 
using Theorem 5 (ii) with $T_{1}$ and $T_{2}$ interchanged. Hence, $\left|\mathcal{E}_{T_{1}}\right|=\left|\mathcal{E}_{T_{2}}\right|$ follows by the existence of such $h$.

Remark 4. Example 3 showed one particular case of two theories $T_{1}$ and $T_{2}$ such that $\left|T_{1}\right| \neq\left|T_{2}\right|$ but $\left|\mathcal{E}_{T_{1}}\right|=\left|\mathcal{E}_{T_{2}}\right|$. Again, in case of general hedges, the previous observations do not hold. As an example, one may take $T_{3}$ and $T_{4}$ from Example 2 considering the three-element Gödel chain with identity as the hedge.

In order to get further insight into the structure of non-redundant theories, we introduce a particular notion of provability which is stronger than the one we have considered so far. The notion is an analog of the direct determination [23] established in the framework of the classic functional dependencies.

Definition 7. Let $T$ be a theory, $A, B \in L^{Y}$. We say that $A \Rightarrow B$ is directly provable by $T$, written $T \Vdash A \Rightarrow B$, whenever

$$
T \backslash \mathrm{E}_{T}(A) \vdash A \Rightarrow B .
$$

Remark 5. Obviously, $\Vdash$ is stronger than $\vdash$. If $A \Rightarrow B$ is trivial then $T \Vdash A \Rightarrow B$ for arbitrary $T$ since for $B \subseteq A$, we have $\emptyset \vdash A \Rightarrow B$. In particular, $T \Vdash A \Rightarrow A$. In general, $\Vdash$ and $\vdash$ do not coincide. For instance, consider $T=\{A \Rightarrow B\}$ where $A \Rightarrow B$ is non-trivial, i.e., $B \nsubseteq A$. In that case, $\mathrm{E}_{T}(A)=\{A \Rightarrow B\}$, i.e., $T \backslash \mathrm{E}_{T}(A) \nvdash A \Rightarrow B$, meaning that $T \nVdash A \Rightarrow B$.

Example 4. Take $T_{2}$ from Example 2 and let $Y=\{x, y, z\}$. The total number of formulas (using $Y$ as the set of all attributes) which are provable by $T_{2}$ is 543 , among those are 327 non-trivial ones. The number of formulas which are directly provable by $T_{2}$ is considerably lower. Namely, only 231 formulas are 
directly provable by $T_{2}$. Moreover, only 15 of them are non-trivial ones. Namely,

$$
\begin{aligned}
& T^{\prime}=\left\{\left\{^{0.5} / y, z\right\} \Rightarrow\left\{{ }^{0.5} / x\right\},\left\{{ }^{0.5} / y, z\right\} \Rightarrow\left\{{ }^{0.5} / x,{ }^{0.5} / z\right\},\{0.5 / y, z\} \Rightarrow\left\{{ }^{0.5} / x, z\right\},\right. \\
& \left\{{ }^{0.5} / y, z\right\} \Rightarrow\left\{{ }^{0.5} / x,{ }^{0.5} / y\right\},\{0.5 / y, z\} \Rightarrow\left\{{ }^{0.5} / x,{ }^{0.5} / y,{ }^{0.5} / z\right\}, \\
& \{0.5 / y, z\} \Rightarrow\left\{0.5 / x,{ }^{0.5} / y, z\right\},\{y, z\} \Rightarrow\{0.5 / x\},\{y, z\} \Rightarrow\left\{{ }^{0.5} / x,{ }^{0.5} / z\right\}, \\
& \{y, z\} \Rightarrow\left\{{ }^{0.5} / x, z\right\},\{y, z\} \Rightarrow\left\{{ }^{0.5} / x,{ }^{0.5} / y\right\},\{y, z\} \Rightarrow\left\{{ }^{0.5} / x,{ }^{0.5} / y,{ }^{0.5} / z\right\}, \\
& \{y, z\} \Rightarrow\left\{{ }^{0.5} / x,{ }^{0.5} / y, z\right\},\{y, z\} \Rightarrow\left\{{ }^{0.5} / x, y\right\},\{y, z\} \Rightarrow\left\{{ }^{0.5} / x, y,{ }^{0.5} / z\right\}, \\
& \{y, z\} \Rightarrow\{0.5 / x, y, z\}\}
\end{aligned}
$$

is the set of all non-trivial formulas which are directly provable by $T_{2}$.

The following assertion shows that theories equivalent in terms of $\vdash$ are also equivalent in terms of $\Vdash$. That means, when considering direct provability, one may replace a theory by an equivalent one.

Theorem 8. Let $T_{1}$ and $T_{2}$ be equivalent. If $T_{1} \Vdash A \Rightarrow B$, then $T_{2} \Vdash A \Rightarrow B$.

Proof. Assume that $T_{1} \Vdash A \Rightarrow B$ and take minimal $S \subseteq T_{1} \backslash \mathrm{E}_{T_{1}}(A)$ such that $S \vdash A \Rightarrow B$, i.e., $A \Rightarrow B$ is not provable by any proper subset of $S$. Now it suffices to show that each formula in $S$ is provable by $T_{2} \backslash \mathrm{E}_{T_{2}}(A)$. Indeed, by $S \vdash A \Rightarrow B$ we then conclude that $A \Rightarrow B$ is provable by $T_{2} \backslash \mathrm{E}_{T_{2}}(A)$.

Thus, take any $C \Rightarrow D \in S$. Since $S \vdash A \Rightarrow B$ and $S \backslash\{C \Rightarrow D\} \nvdash A \Rightarrow B$, which is a consequence of the minimality of $S$, Theorem 2 gives $S \vdash A \Rightarrow C$. That is, $T_{1} \vdash A \Rightarrow C$ on account of $S \subseteq T_{1}$.

By contradiction, assume that $C \Rightarrow D$ is not provable by $T_{2} \backslash \mathrm{E}_{T_{2}}(A)$. Since it is obviously provable by $T_{2}$ ( $T_{2}$ is equivalent to $T_{1}$ and $S \subseteq T_{1}$ ), it means that each proof of $C \Rightarrow D$ by $T_{2}$ contains a formula in $\mathrm{E}_{T_{2}}(A)$. Let $R$ be a minimal subset of $T_{2}$ such that $R \vdash C \Rightarrow D$. By the minimality of $R$ and utilizing the fact that each proof of $C \Rightarrow D$ by $R$ contains a formula in $\mathrm{E}_{T_{2}}(A)$, it follows that there is $G \Rightarrow H \in \mathrm{E}_{T_{2}}(A)$ such that $R \backslash\{G \Rightarrow H\} \nvdash C \Rightarrow D$. By Theorem 2, we get $R \vdash C \Rightarrow G$ which further gives $T_{2} \vdash C \Rightarrow G$. Moreover, $G \Rightarrow H \in \mathrm{E}_{T_{2}}(A)$ gives $T_{2} \vdash G \Rightarrow A$. Hence, by $T_{2} \vdash C \Rightarrow G$ and $T_{2} \vdash G \Rightarrow A$, we get $T_{2} \vdash C \Rightarrow A$, i.e., $T_{1} \vdash C \Rightarrow A$. Taking into account the assumption 
$T_{1} \vdash A \Rightarrow C$ from the previous paragraph, we conclude that $A \equiv_{T_{1}} C$. The latter observation means that $C \Rightarrow D \in \mathrm{E}_{T_{1}}(A)$ which contradicts the fact that $C \Rightarrow D \in S \subseteq T_{1} \backslash \mathrm{E}_{T_{1}}(A)$.

Corollary 9. Let $T_{1}$ and $T_{2}$ be equivalent. Then, for any $A, B \in L^{Y}$, we have $T_{1} \Vdash A \Rightarrow B$ iff $T_{2} \Vdash A \Rightarrow B$.

For any theory $T$, it is easily seen that by $T \vdash A \Rightarrow B$ and $T \vdash B \Rightarrow C$ one can infer $T \vdash A \Rightarrow C$. This is an immediate consequence of applying (Tra). An analogous rule of transitivity can also be proved in case of $\Vdash$ :

Lemma 10. If $T \Vdash A \Rightarrow B$, and $T \Vdash B \Rightarrow C$, then $T \Vdash A \Rightarrow C$.

Proof. Clearly, the claim is trivial if $B \Rightarrow C$ is a trivial formula, i.e., if $C \subseteq B$. Assume that $B \Rightarrow C$ is non-trivial. Observe that if $T \backslash \mathrm{E}_{T}(A) \vdash B \Rightarrow C$, the claim follows directly by (Tra). So, it suffices to show that $B \Rightarrow C$ is always provable by $T \backslash \mathrm{E}_{T}(A)$. By way of contradiction, assume that $T \backslash \mathrm{E}_{T}(A) \nvdash$ $B \Rightarrow C$. Since $T \vdash B \Rightarrow C$, there are $T^{\prime}$ and $D \Rightarrow E \in \mathrm{E}_{T}(A)$ such that $T \backslash \mathrm{E}_{T}(A) \subset T^{\prime} \subseteq T, T^{\prime} \vdash B \Rightarrow C$, and $T^{\prime} \backslash\{D \Rightarrow E\} \nvdash B \Rightarrow C$. Using Theorem 2, it follows that $T^{\prime} \backslash\{D \Rightarrow E\} \vdash B \Rightarrow D$ and so $T \vdash B \Rightarrow A$ using (Tra) and the monotony of provability together with the fact that $T \vdash D \Rightarrow A$. In addition, using $T \vdash A \Rightarrow B$, we get $A \equiv_{T} B$, i.e., $\mathrm{E}_{T}(A)=\mathrm{E}_{T}(B)$ which contradicts our assumption $T \backslash \mathrm{E}_{T}(A) \nvdash B \Rightarrow C$ because $T \Vdash B \Rightarrow C$ means $T \backslash \mathrm{E}_{T}(B) \vdash B \Rightarrow C$.

In the following assertions, we explore antecedents of formulas in $\mathrm{E}_{T}(A)$. Therefore, for any $A \in L^{Y}$, we put

$$
\mathrm{e}_{T}(A)=\left\{C \in L^{Y} \mid C \Rightarrow D \in \mathrm{E}_{T}(A)\right\} .
$$

As in case of $\mathrm{E}_{T}(A)$, we may have $\mathrm{e}_{T}(A)=\emptyset$.

Theorem 11. Let $T$ be a theory and let $\mathrm{e}_{T}(A) \neq \emptyset$. For each $C \in L^{Y}$ satisfying $A \equiv_{T} C$ there is $D \in \mathrm{e}_{T}(A)$ such that $T \Vdash C \Rightarrow D$. 
Proof. Take arbitrary $C \in L^{Y}$ such that $A \equiv_{T} C$. For every $G \in \mathrm{e}_{T}(A)$ we get $C \equiv_{T} G$ and thus $T \vdash C \Rightarrow G$. Take $T^{\prime} \subseteq T$ and $D \in \mathrm{e}_{T}(A)$ with the following property: $T^{\prime} \vdash C \Rightarrow D$ and if $T^{\prime \prime} \vdash C \Rightarrow G$ for $T^{\prime \prime} \subseteq T$ and $G \in \mathrm{e}_{T}(A)$, then $\left|T^{\prime}\right| \leq\left|T^{\prime \prime}\right|$. Thus, $T^{\prime}$ has the minimal size among all theories which prove some formula of the form $C \Rightarrow G$, where $G \in \mathrm{e}_{T}(A)$. We now show, that $T^{\prime} \cap \mathrm{E}_{T}(A)=\emptyset$ by which we get $T \backslash \mathrm{E}_{T}(A) \vdash C \Rightarrow D$ yielding $T \Vdash C \Rightarrow D$.

By way of contradiction, let $G \Rightarrow H \in T^{\prime}$ and $G \Rightarrow H \in \mathrm{E}_{T}(A)$. Hence, $G \in \mathrm{e}_{T}(A)$ and using the minimality of $T^{\prime}$, we get $T^{\prime} \backslash\{G \Rightarrow H\} \nvdash C \Rightarrow D$. Applying Theorem 2, we get $T^{\prime} \backslash\{G \Rightarrow H\} \vdash C \Rightarrow G$, i.e., $T^{\prime} \backslash\{G \Rightarrow H\}$ contains less formulas than $T^{\prime}$ and proves $C \Rightarrow G$ with $G \in \mathrm{e}_{T}(A)$, contradicting the minimality of $T^{\prime}$.

Example 5. We show non-trivial applications of Theorem 11 Consider $T_{2}$ from Example 2 Take $\{0.5 / x, y, 0.5 / z\} \Rightarrow\{x, 0.5 / y, 0.5 / z\} \in T_{2}$ and let $C=\{0.5 / y, z\}$. Then, for $D=\left\{0.5 / x,{ }^{0.5} / y\right\}$, we have $T_{2} \Vdash C \Rightarrow D$. In a more detail, we have

$$
T_{2} \backslash \mathrm{E}_{T_{2}}(C)=\{\{z\} \Rightarrow\{0.5 / x\}\},
$$

cf. Example 3. In addition, (Cut) applied to $\left\{{ }^{0.5} / y, z\right\} \Rightarrow\{z\}$ and $\{z\} \Rightarrow\{0.5 / x\}$ yields $\left\{{ }^{0.5} / y, z\right\} \Rightarrow\{0.5 / x\}$ and thus $\{0.5 / y, z\} \Rightarrow\left\{^{0.5} / x, 0.5 / y\right\}$ is provable by $T_{2} \backslash \mathrm{E}_{T_{2}}(C)$, showing $T_{2} \Vdash C \Rightarrow D$. Analogously, for $C=\{y, z\}$, we may take $D=\left\{0.5 / x, y,{ }^{0.5} / z\right\}$ or $D=\left\{0.5 / x,{ }^{0.5} / y\right\}$ and have $T_{2} \Vdash C \Rightarrow D$.

The following assertion is used in the process of finding minimal theories. It shows that under conditions formulated by equivalence and direct provability, a pair of formulas in a theory can be equivalently replaced by a single formula.

Theorem 12. Let $T$ be a theory such that for $A \Rightarrow B \in T$ and $C \Rightarrow D \in T$, we have $A \equiv_{T} C$ and $T \Vdash A \Rightarrow C$. Then,

$$
(T \backslash\{A \Rightarrow B, C \Rightarrow D\}) \cup\{C \Rightarrow B \cup D\}
$$

is equivalent to $T$. 
Proof. Denote the theory (13) by $T^{\prime}$. Since $A \Rightarrow B \in T$, we get $T \vdash A \Rightarrow B$. Furthermore, $T \vdash C \Rightarrow A$ because $A \equiv_{T} C$. Therefore, by (Tra), we get $T \vdash C \Rightarrow B$. Moreover, using the fact that $C \Rightarrow D \in T$ and applying (Add), we get $T \vdash C \Rightarrow B \cup D$.

Conversely, it suffices to show that both $A \Rightarrow B$ and $C \Rightarrow D$ are provable by $T^{\prime}$. Clearly, $T^{\prime} \vdash C \Rightarrow D$ results by $C \Rightarrow B \cup D \in T^{\prime}$ using (Pro). In order to see that $A \Rightarrow B$ is provable by $T^{\prime}$, observe first that $T^{\prime} \vdash A \Rightarrow C$. Indeed, $T \Vdash A \Rightarrow C$ means that $T \backslash \mathrm{E}_{T}(A) \vdash A \Rightarrow C$. Therefore, taking into account $A \equiv_{T} C$, we get $A \Rightarrow B \notin T \backslash \mathrm{E}_{T}(A)$ and $C \Rightarrow D \notin T \backslash \mathrm{E}_{T}(A)$, showing $T \backslash\{A \Rightarrow B, C \Rightarrow D\} \vdash A \Rightarrow C$ which further gives $T^{\prime} \vdash A \Rightarrow C$. Now, using $T^{\prime} \vdash C \Rightarrow B \cup D$ and (Tra), we obtain $T^{\prime} \vdash A \Rightarrow B \cup D$ and consequently $T^{\prime} \vdash A \Rightarrow B$ by (Pro).

By a particular application of Theorem 12 , we may find an equivalent theory which consists of formulas with modified antecedents:

Corollary 13. Let $T$ be a theory such that $A \Rightarrow B \in T, A \equiv_{T} C$, and $T \Vdash$ $A \Rightarrow C$. Then, $(T \backslash\{A \Rightarrow B\}) \cup\{C \Rightarrow B\}$ is equivalent to $T$.

Proof. Take $T^{\prime}=T \cup\{C \Rightarrow C\}$. By Theorem 12, $T^{\prime}$ is equivalent to

$$
\left(T^{\prime} \backslash\{A \Rightarrow B, C \Rightarrow C\}\right) \cup\{C \Rightarrow B \cup C\}=(T \backslash\{A \Rightarrow B\}) \cup\{C \Rightarrow B \cup C\},
$$

which is equivalent to $(T \backslash\{A \Rightarrow B\}) \cup\{C \Rightarrow B\}$ because $\{C \Rightarrow B \cup C\} \vdash C \Rightarrow B$ by (Pro) and $\{C \Rightarrow B\} \vdash C \Rightarrow B \cup C$ by the axiom $C \Rightarrow C$ and (Add).

Example 6. Considering $T_{2}$ from Example 2, there are three pairs of formulas $A \Rightarrow B \in T_{2}$ and $C \Rightarrow D \in T_{2}$ satisfying the conditions of Theorem 12 and which in turn can be used to find a theory which is equivalent to $T_{2}$ and is strictly smaller. Namely, we may

- use $\left\{{ }^{0.5} / x, y,{ }^{0.5} / z\right\} \Rightarrow\left\{x,{ }^{0.5} / y,{ }^{0.5} / z\right\}$ and $\left\{{ }^{0.5} / y\right\} \Rightarrow\left\{{ }^{0.5} / x,{ }^{0.5} / y, z\right\}$, and replace the formulas by $\{0.5 / y\} \Rightarrow\left\{x,{ }^{0.5} / y, z\right\}$; or

- use $\{0.5 / x, y, 0.5 / z\} \Rightarrow\{x, 0.5 / y, 0.5 / z\}$ and $\left\{0.5 / x,{ }^{0.5} / y\right\} \Rightarrow\{y, z\}$, and replace the formulas by $\left\{0.5 / x,{ }^{0.5} / y\right\} \Rightarrow\{x, y, z\}$; or 
- use $\left\{0.5 / x,{ }^{0.5} / y\right\} \Rightarrow\{y, z\}$ and $\{0.5 / y\} \Rightarrow\{0.5 / x, 0.5 / y, z\}$,

and replace the formulas by $\{0.5 / y\} \Rightarrow\{0.5 / x, y, z\}$.

Lemma 14. Let $T_{1}$ and $T_{2}$ be equivalent and non-redundant. Then, for each $A \in \mathrm{e}_{T_{1}}(H)$ there is $C \in \mathrm{e}_{T_{2}}(H)$ such that $T_{1} \Vdash A \Rightarrow C$.

Proof. Observe that by $A \in \mathrm{e}_{T_{1}}(H)$ and Theorem 4 it follows that $\mathrm{e}_{T_{2}}(H) \neq \emptyset$, i.e., there is $C^{\prime} \Rightarrow D^{\prime} \in T_{2}$ such that $C^{\prime} \equiv_{T_{2}} H$ and thus $C^{\prime} \equiv_{T_{2}} A$. Using Theorem 11. there is $C \in \mathrm{e}_{T_{2}}\left(C^{\prime}\right)=\mathrm{e}_{T_{2}}(H)$ such that $T_{2} \Vdash A \Rightarrow C$. Since $T_{1}$ and $T_{2}$ are equivalent, $T_{1} \Vdash A \Rightarrow C$ by Theorem 8 .

Example 7. We illustrate the correspondence between antecedents of formulas from Lemma 14. Considering theories $T_{1}$ and $T_{2}$ from Example 2 for each $A \in \mathrm{e}_{T_{1}}(H)$ there is $C \in \mathrm{e}_{T_{2}}(H)$ such that $T_{1} \Vdash A \Rightarrow C$ because all antecedents of formulas in $T_{1}$ are among the antecendents of formulas in $T_{2}$. Conversely, for $\left\{0.5 / x, y,{ }^{0.5} / z\right\} \Rightarrow\left\{x,{ }^{0.5} / y,{ }^{0.5} / z\right\} \in T_{2}$, we can take $\{0.5 / y\} \Rightarrow$ $\left\{x,{ }^{0.5} / y, z\right\} \in T_{1}$ and obviously $T_{2} \Vdash\{0.5 / x, y, 0.5 / z\} \Rightarrow\{0.5 / y\}$. Analogously, for $\left\{0.5 / x,{ }^{0.5} / y\right\} \Rightarrow\{y, z\} \in T_{2}$ there is $\left\{{ }^{0.5} / y\right\} \Rightarrow\left\{x,{ }^{0.5} / y, z\right\} \in T_{1}$ satisfying $T_{2} \Vdash\left\{{ }^{0.5} / x,{ }^{0.5} / y\right\} \Rightarrow\left\{{ }^{0.5} / y\right\}$. Also note that Corollary 13 allows us to modify theories while preserving their equivalence. For instance, due to our previous observations, $\{0.5 / x, y, 0.5 / z\} \Rightarrow\left\{x,{ }^{0.5} / y,{ }^{0.5} / z\right\} \in T_{2}$ can equivalently be replaced by $\{0.5 / y\} \Rightarrow\{x, 0.5 / y, 0.5 / z\}$.

We now turn our attention to minimal theories, i.e., theories which are minimal in terms of the number of formulas:

Definition 15. A theory $T$ is called minimal (in the number of formulas) if for each equivalent theory $T^{\prime}$, we have $|T| \leq\left|T^{\prime}\right|$.

Obviously, a minimal theory is non-redundant but the converse does not hold in general. Applying Theorem 12, we have the following corollary.

Corollary 16. Let $T$ be minimal. Then there are no distinct $A \Rightarrow B \in T$ and $C \Rightarrow D \in T$ such that $A \equiv_{T} C$ and $T \Vdash A \Rightarrow C$. 
The following assertions shows properties of direct provability by minimal theories and their consequences.

Lemma 17. Let $T_{1}$ and $T_{2}$ be equivalent and minimal. Then for $A, A_{1}, A_{2} \in$ $\mathrm{e}_{T_{1}}(H)$ and $C, C_{1}, C_{2} \in \mathrm{e}_{T_{2}}(H)$, the following conditions hold:

(i) If $T_{1} \Vdash A \Rightarrow C$, then $T_{1} \Vdash C \Rightarrow A$.

(ii) If $T_{1} \Vdash A \Rightarrow C_{1}$ and $T_{1} \Vdash A \Rightarrow C_{2}$, then $C_{1}=C_{2}$.

(iii) If $T_{1} \Vdash A_{1} \Rightarrow C$ and $T_{1} \Vdash A_{2} \Rightarrow C$, then $A_{1}=A_{2}$.

Proof. In order to prove (i), we use Lemma 14 to conclude that for $C \in \mathrm{e}_{T_{2}}(H)$ there is $A^{\prime} \in \mathrm{e}_{T_{1}}(H)$ such that $T_{2} \Vdash C \Rightarrow A^{\prime}$, i.e., $T_{1} \Vdash C \Rightarrow A^{\prime}$. Now, using the assumption $T_{1} \Vdash A \Rightarrow C$, Lemma 10 yields $T_{1} \Vdash A \Rightarrow A^{\prime}$. In addition to that, there are $A \Rightarrow B \in T_{1}$ and $A^{\prime} \Rightarrow B^{\prime} \in T_{1}$ with $A \equiv_{T} A^{\prime}$. Hence, by Corollary 16, we get that $A=A^{\prime}$, meaning that $T_{1} \Vdash C \Rightarrow A$.

In case of (ii), we proceed analogously as in (i). By $T_{1} \Vdash A \Rightarrow C_{1}$, we get $T_{1} \Vdash C_{1} \Rightarrow A$ by (i) and thus $T_{1} \Vdash C_{1} \Rightarrow C_{2}$ by Lemma 10 . Then, Corollary 16 yields $C_{1}=C_{2}$.

Finally, by $T_{1} \Vdash A_{2} \Rightarrow C$ it follows $T_{1} \Vdash C \Rightarrow A_{2}$ by (i). So, analogously as in case of (ii), Lemma 10 and Corollary 16 imply $T_{1} \Vdash A_{1} \Rightarrow A_{2}$ and thus $A_{1}=A_{2}$, which proves (iii).

Theorem 18. Let $T_{1}$ and $T_{2}$ be equivalent and minimal. Then, for each $H \in$ $L^{Y}$, there is an injective map $h_{H}: \mathrm{e}_{T_{1}}(H) \rightarrow \mathrm{e}_{T_{2}}(H)$. Furthermore, $\left|\mathrm{e}_{T_{1}}(H)\right|=$ $\left|\mathrm{e}_{T_{2}}(H)\right|$.

Proof. If $\mathrm{e}_{T_{1}}(H)$ is non-empty, then using Lemma 14 and Lemma 17(ii) it follows that $h_{H}$ can be defined by $h_{H}(A)=C$, where $T_{1} \Vdash A \Rightarrow C$. In addition, Lemma 17 (iii) gives that $h_{H}$ is injective. Thus, $\left|\mathrm{e}_{T_{1}}(H)\right| \leq\left|\mathrm{e}_{T_{2}}(H)\right|$. The second part follows by application of the claim with $T_{1}$ and $T_{2}$ interchanged.

Finally, the next theorem shows that in case of non-redundant theories which are not minimal, one can always transform the non-redundant theory into an 
equivalent and smaller one because the theory contains formulas satisfying the assumption of Theorem 12 .

Theorem 19. Let $T$ be non-redundant and not minimal. Then, there are distinct formulas $A \Rightarrow B \in T$ and $C \Rightarrow D \in T$ such that $A \equiv_{T} C$ and $T \Vdash A \Rightarrow C$.

Proof. First, observe that if there are $A \Rightarrow B \in T$ and $C \Rightarrow D \in T$ such that $A=C$, then trivially $A \equiv_{T} C$ and $T \Vdash A \Rightarrow C$. So, assume that $T$ contains no such formulas. Taking into account this assumption, we have $\left|\mathrm{E}_{T}(H)\right|=\left|\mathrm{e}_{T}(H)\right|$ for all $H \in L^{Y}$. Furthermore, let $T^{\prime}$ be a minimal theory which is equivalent to $T$. Since it is minimal, we also have $\left|\mathrm{e}_{T^{\prime}}(H)\right|=\left|\mathrm{E}_{T^{\prime}}(H)\right|$ for all $H \in L^{Y}$.

Since $T$ is not minimal, then there is $H \in L^{Y}$ such that $\left|\mathrm{e}_{T^{\prime}}(H)\right|=\left|\mathrm{E}_{T^{\prime}}(H)\right|<$ $\left|\mathrm{E}_{T}(H)\right|=\left|\mathrm{e}_{T}(H)\right|$ because otherwise the non-minimality of $T$ would be violated. Using Lemma 14, for any $A_{i} \in \mathrm{e}_{T}(H)$ there is $C_{i} \in \mathrm{e}_{T^{\prime}}(H)$ such that $T \Vdash A_{i} \Rightarrow C_{i}$. Considering $\left|\mathrm{e}_{T^{\prime}}(H)\right|<\left|\mathrm{e}_{T}(H)\right|$ and using the pigeonhole principle, there are $A_{1}, A_{2} \in \mathrm{e}_{T}(H)$ and $C \in \mathrm{e}_{T^{\prime}}(H)$ such that $A_{1} \neq A_{2}, T \Vdash A_{1} \Rightarrow C$, and $T \Vdash A_{2} \Rightarrow C$. Moreover, for $C$ there is $A^{\prime} \in \mathrm{e}_{T}(H)$ such that $T^{\prime} \Vdash C \Rightarrow A^{\prime}$, i.e., $T \Vdash C \Rightarrow A^{\prime}$. Since $A_{1}$ and $A_{2}$ are distinct, either $A_{1} \neq A^{\prime}$ or $A_{2} \neq A^{\prime}$. In case of $A_{1} \neq A^{\prime}$, the fact that $A_{1}, A^{\prime} \in \mathrm{e}_{T}(H)$ yields that $A_{1} \equiv_{T} A$ and there are $A_{1} \Rightarrow B \in T$ and $A^{\prime} \Rightarrow D \in T$. Furthermore, by $T \Vdash A_{1} \Rightarrow C$ and $T \Vdash C \Rightarrow A^{\prime}$ it follows that $T \Vdash A_{1} \Rightarrow A^{\prime}$ by Lemma 10 Thus, the desired formulas we look for are $A_{1} \Rightarrow B \in T$ and $A^{\prime} \Rightarrow D \in T$. The case of $A_{2} \neq A^{\prime}$ uses the same arguments.

Based on our observations, we may introduce an algorithm which, given a theory $T$, finds a theory which is equivalent to $T$ and minimal. Indeed, one may utilize a standard procedure to find a non-redundant subset of $T$. That is, one removes all $A \Rightarrow B \in T$ such that $T \backslash\{A \Rightarrow B\} \vdash A \Rightarrow B$. Then, Corollary 16 and Theorem 19 yield an if and only if condition for $T$ being minimal. Namely, $T$ is minimal iff it does not contain distinct $A \Rightarrow B \in T$ and $C \Rightarrow D \in T$ such that $A \equiv_{T} C$ and $T \Vdash A \Rightarrow C$. If $T$ contains such formulas, one applies Theorem 12 to find a theory which is strictly smaller than $T$. Then, one may 
repeat the process until the theory is minimal. The algorithm is illustrated by the following example.

Example 8. We conclude the examples by applying the previous observations to find a minimal theory which is equivalent to $T_{2}$ from Example 2. Recall that $T_{2}$ is non-redundant but it is not minimal ( $T_{1}$ from Example 2 is equivalent to $T_{2}$ and is strictly smaller). Since $T_{2}$ is not minimal, Theorem 19 ensures there are $A \Rightarrow B \in T_{2}$ and $C \Rightarrow D \in T_{2}$ such that $A \equiv_{T_{2}} C$ and $T_{2} \Vdash A \Rightarrow$ $C$. In particular, we may take $\left\{0.5 / x, y,{ }^{0.5} / z\right\} \Rightarrow\left\{x,{ }^{0.5} / y, 0.5 / z\right\}$ for $A \Rightarrow B$ and $\{0.5 / y\} \Rightarrow\left\{{ }^{0.5} / x,{ }^{0.5} / y, z\right\}$ for $C \Rightarrow D$. Applying Theorem $12, T_{2}$ can be transformed into $T_{2}^{\prime}$ of the form

$$
\begin{aligned}
& T_{2}^{\prime}=\left\{\left\{^{0.5} / y\right\} \Rightarrow\left\{x,{ }^{0.5} / y, z\right\},\{z\} \Rightarrow\left\{{ }^{0.5} / x\right\},\right. \\
&\left.\left\{{ }^{0.5} / x,{ }^{0.5} / y\right\} \Rightarrow\{y, z\},\{x, z\} \Rightarrow\left\{{ }^{0.5} / x, y,{ }^{0.5} / z\right\}\right\}
\end{aligned}
$$

which is strictly smaller than $T_{2}$. Applying Corollary $16, T_{2}^{\prime}$ is not minimal since for $A \Rightarrow B$ being $\left.\left\{{ }^{0.5} / x,{ }^{0.5} / y\right\} \Rightarrow\{y, z\}\right\}$ and for $C \Rightarrow D$ being $\{0.5 / y\} \Rightarrow$ $\left\{x,{ }^{0.5} / y, z\right\}$, we have $A \equiv_{T_{2}^{\prime}} C$ and $T_{2}^{\prime} \Vdash A \Rightarrow C$. Therefore, we may apply Theorem 12 in order to transform $T_{2}^{\prime}$ into

$$
T_{2}^{\prime \prime}=\left\{\left\{^{0.5} / y\right\} \Rightarrow\{x, y, z\},\{z\} \Rightarrow\{.5 / x\},\{x, z\} \Rightarrow\left\{^{0.5} / x, y,{ }^{0.5} / z\right\}\right\} .
$$

As one can check, $T_{2}^{\prime \prime}$ contains no distinct $A \Rightarrow B$ and $C \Rightarrow D$ such that $A \equiv_{T_{2}^{\prime \prime}} C$ and $T_{2}^{\prime \prime} \Vdash A \Rightarrow C$. Hence, by Theorem 19. $T_{2}^{\prime \prime}$ is minimal. Notice that we have derived a minimal equivalent theory $T_{2}^{\prime \prime}$ from $T_{2}$ without using $T_{1}$ (from Example 21. Also, the minimal equivalent theories $T_{2}^{\prime \prime}$ and $T_{1}$ are distinct.

Remark 6 . The the asymptotic time complexity of obtaining a minimal equivalent theory is polynomial. Indeed, given a theory $T$, Theorem 19 is applied at most $|T|$ times. In each step, we inspect pairs of formulas $A \Rightarrow B$ and $C \Rightarrow D$ such that $A \equiv_{T} C$ and $T \Vdash A \Rightarrow C$. Both $A \equiv_{T} C$ and $T \Vdash A \Rightarrow C$ can be tested based on computing closures, i.e., in time $O(n l)$, where $n$ is the length of $T$ (total number of attributes appearing in all formulas in $T$ ) and $l$ is the size of the structure of degrees (i.e., $l$ is a multiplicative constant depending 
on $\mathbf{L}$ ), see GLinClosure [6]. Interestingly, the information on equivalence of $\mathbf{L}$-sets of attributes and on direct provability can be computed only once. Indeed, since the algorithm transforms theories into equivalent ones, by Theorem 8 , we get that the direct provability of formulas is preserved. This makes testing of $A \equiv_{T} C$ and $T \Vdash A \Rightarrow C$ efficient. Altogether, the algorithm runs in $O\left(n^{2} l\right)$, where $n$ is the length of $T$, and $l$ is the size of $\mathbf{L}$. This is in contrast with the instance-based approach mentioned in the introduction which relies on computing pseudo-intents which is hard even in the bivalent case, see [10.

\section{Conclusion}

We presented an if-and-only-if criterion of minimality of non-redundant set of graded attribute implications with semantics parameterized by globalization. The result is constructive and allows to transform a non-redundant set of graded attribute implications into an equivalent and minimal one. Issues which we find interesting for future research include generalization of the approach to accomodate arbitrary linguistic hedges, construction of efficient algorithms based on the present result, and connections to other techniques for removing redundancy in both the classic and graded settings, e.g., the instance-based approaches like [17, 34.

\section{Acknowledgment}

Supported by grant no. P202/14-11585S of the Czech Science Foundation.

\section{References}

[1] Rakesh Agrawal, Tomasz Imieliński, and Arun Swami, Mining association rules between sets of items in large databases, Proceedings of the 1993 ACM SIGMOD International Conference on Management of Data (New York, NY, USA), SIGMOD '93, ACM, 1993, pp. 207-216.

[2] William Ward Armstrong, Dependency structures of data base relationships, Information Processing 74: Proceedings of IFIP Congress (Amsterdam) (J. L. Rosenfeld and H. Freeman, eds.), North Holland, 1974, pp. 580-583. 
[3] Mathias Baaz, Infinite-valued Gödel logics with 0-1 projections and relativizations, GÖDEL '96, Logical Foundations of Mathematics, Computer Sciences and Physics (Berlin/Heidelberg), Lecture Notes in Logic, vol. 6, Springer-Verlag, 1996, pp. 23-33.

[4] Radim Belohlavek, Fuzzy Relational Systems: Foundations and Principles, Kluwer Academic Publishers, Norwell, MA, USA, 2002.

[5] Radim Belohlavek and Vilem Vychodil, Data tables with similarity relations: Functional dependencies, complete rules and non-redundant bases, Database Systems for Advanced Applications (Mong Lee, Kian-Lee Tan, and Vilas Wuwongse, eds.), Lecture Notes in Computer Science, vol. 3882, Springer Berlin Heidelberg, 2006, pp. 644-658.

[6] _ Basic algorithm for attribute implications and functional dependencies in graded setting, International Journal of Foundations of Computer Science 19 (2008), no. 2, 297317.

[7] _ Formal concept analysis and linguistic hedges, International Journal of General Systems 41 (2012), no. 5, 503-532.

[8] _ Attribute dependencies for data with grades, CoRR abs/1402.2071 (2014), http://arxiv.org/abs/1402.2071

[9] Pablo Cordero, Ángel. Mora, Inmaculada Pérez de Guzmán, and Manuel Enciso, Nondeterministic ideal operators: An adequate tool for formalization in data bases, Discrete Applied Mathematics 156 (2008), no. 6, 911-923.

[10] Felix Distel and Barıs Sertkaya, On the complexity of enumerating pseudo-intents, Discrete Appl. Math. 159 (2011), no. 6, 450-466.

[11] Francesc Esteva and Lluís Godo, Monoidal t-norm based logic: Towards a logic for leftcontinuous t-norms, Fuzzy Sets and Systems 124 (2001), no. 3, 271-288.

[12] Francesc Esteva, Lluís Godo, and Carles Noguera, A logical approach to fuzzy truth hedges, Information Sciences 232 (2013), 366-385.

[13] Nikolaos Galatos, Peter Jipsen, Tomacz Kowalski, and Hiroakira Ono, Residuated Lattices: An Algebraic Glimpse at Substructural Logics, Volume 151, 1st ed., Elsevier Science, San Diego, USA, 2007.

[14] Bernhard Ganter and Rudolf Wille, Formal concept analysis: Mathematical foundations, 1st ed., Springer-Verlag New York, Inc., Secaucus, NJ, USA, 1997.

[15] Joseph A. Goguen, The logic of inexact concepts, Synthese 19 (1979), 325-373.

[16] Siegfried Gottwald, Mathematical fuzzy logics, Bulletin of Symbolic Logic 14 (2008), no. 2, 210-239.

[17] Jean-Louis Guigues and Vincent Duquenne, Familles minimales d'implications informatives resultant d'un tableau de données binaires, Math. Sci. Humaines 95 (1986), 5-18. 
[18] Petr Hájek, Metamathematics of Fuzzy Logic, Kluwer Academic Publishers, Dordrecht, The Netherlands, 1998.

[19] _ On very true, Fuzzy Sets and Systems 124 (2001), no. 3, 329-333.

[20] Ulrich Höhle, Monoidal logic, Fuzzy-Systems in Computer Science (R. Kruse, J. Gebhardt, and R. Palm, eds.), Artificial Intelligence / Künstliche Intelligenz, Vieweg+Teubner Verlag, 1994, pp. 233-243.

[21] Richard Holzer, Knowledge acquisition under incomplete knowledge using methods from formal concept analysis: Part I, Fundamenta Informaticae 63 (2004), no. 1, 17-39.

[22] John W. Lloyd, Foundations of Logic Programming, Springer-Verlag New York, Inc., New York, NY, USA, 1984.

[23] David Maier, Theory of Relational Databases, Computer Science Pr, Rockville, MD, USA, 1983.

[24] Jan Pavelka, On fuzzy logic I: Many-valued rules of inference, Mathematical Logic Quarterly 25 (1979), no. 3-6, 45-52.

[25] _ On fuzzy logic II: Enriched residuated lattices and semantics of propositional calculi, Mathematical Logic Quarterly 25 (1979), no. 7-12, 119-134.

[26] _ On fuzzy logic III: Semantical completeness of some many-valued propositional calculi, Mathematical Logic Quarterly 25 (1979), no. 25-29, 447-464.

[27] Gaisi Takeuti and Satoko Titani, Globalization of intuitionistic set theory, Annals of Pure and Applied Logic 33 (1987), 195-211.

[28] Lucie Urbanova and Vilem Vychodil, Derivation digraphs for dependencies in ordinal and similarity-based data, Information Sciences 268 (2014), 381-396.

[29] Lotfi A. Zadeh, A fuzzy-set-theoretic interpretation of linguistic hedges, Journal of Cybernetics 2 (1972), no. 3, 4-34.

[30] - The concept of a linguistic variable and its application to approximate reasoning-I, Information Sciences 8 (1975), no. 3, 199-249.

[31] _ The concept of a linguistic variable and its application to approximate reasoning-II, Information Sciences 8 (1975), no. 4, 301-357.

[32] _ - The concept of a linguistic variable and its application to approximate reasoning-III, Information Sciences 9 (1975), no. 1, 43-80.

[33] Mohammed J. Zaki, Mining non-redundant association rules, Data Mining and Knowledge Discovery 9 (2004), 223-248.

[34] Yanhui Zhai, Deyu Li, and Kaishe Qu, Decision implication canonical basis: a logical perspective, Journal of Computer and System Sciences (2014),

http://dx.doi.org/10.1016/j.jcss.2014.06.001 